\title{
Estimation of subepithelial lateral extent in submucosal early gastric cancer: retrospective histological analysis
}

\author{
Osamu Goto $\cdot$ Ai Fujimoto $\cdot$ Masayuki Shimoda $\cdot$ Yasutoshi Ochiai $\cdot$ \\ Tatsuo Matsuda $\cdot$ Hiroya Takeuchi $\cdot$ Hirofumi Kawakubo $\cdot$ Tadateru Maehata \\ Toshio Uraoka $\cdot$ Kaori Kameyama $\cdot$ Yuko Kitagawa $\cdot$ Naohisa Yahagi
}

Received: 1 July 2014/ Accepted: 26 August 2014/Published online: 21 October 2014

(C) The International Gastric Cancer Association and The Japanese Gastric Cancer Association 2014

\begin{abstract}
Background Endoscopic full-thickness resection (EFTR) is expected to make possible minimally invasive local resection of early gastric cancer (EGC). However, no consensus exists regarding how far an optimal safety margin should be set in determining the resection area by endoscopy. We aimed to investigate the optimal lateral margin of EGC which could be a candidate for EFTR by measuring the subepithelial extent (SE) of tumors.

Methods In 60 surgically resected submucosal EGCs $4 \mathrm{~cm}$ or smaller, 595 prepared slides which showed lateral tumor borders both on the epithelial surface and on the subepithelial layer were assessed. The distance between the epithelial and the subepithelial tumor edge was measured under microscopic observation, followed by analyses of the relationship between the measured SE and the histological characteristics.

Results The average and the median SE were $1.1 \mathrm{~mm}$ (standard deviation $1.8 \mathrm{~mm}$ ) and $0.3 \mathrm{~mm}$ (range

O. Goto $\cdot$ A. Fujimoto $\cdot$ Y. Ochiai $\cdot$ T. Maehata $\cdot$ T. Uraoka $\cdot$

N. Yahagi $(\bowtie)$

Division of Research and Development for Minimally Invasive Treatment, Cancer Center, School of Medicine, Keio University, 35 Shinanomachi, Shinjuku-ku, Tokyo 160-8582, Japan

e-mail: yahagi.keio@gmail.com

M. Shimoda

Department of Pathology, School of Medicine, Keio University,

Tokyo, Japan

T. Matsuda $\cdot$ H. Takeuchi $\cdot$ H. Kawakubo $\cdot$ Y. Kitagawa Department of Surgery, School of Medicine, Keio University, Tokyo, Japan

K. Kameyama

Division of Diagnostic Pathology, School of Medicine,

Keio University, Tokyo, Japan
\end{abstract}

0-12.3 mm), respectively. The 99th percentile was $8.8 \mathrm{~mm}$. With regard to the histological type, the median SE was significantly greater in diffuse-type tumor than in intestinal-type tumor $(0.9 \mathrm{~mm}$ vs $0 \mathrm{~mm}, p<0.0001)$. With regard to the location of the subepithelilal tumor edge, the median SE was significantly greater in the submucosal layer than in the mucosal layer $(2.6 \mathrm{~mm}$ vs $0.3 \mathrm{~mm}$, $p<0.0001)$.

Conclusions In most lesions, the SE was less than $1 \mathrm{~cm}$. A safety margin may be set at $1 \mathrm{~cm}$ in EFTR of submucosal EGC.

Keywords Early gastric cancer - Endoscopic full-thickness resection - Subepithelial extent

\section{Introduction}

Recent advances in therapeutic endoscopy have raised the possibility of less-invasive gastrectomy by the endoluminal approach. Endoscopic full-thickness resection (EFTR) with or without laparoscopic cooperation has been successfully introduced as a promising technique for resection of gastric submucosal tumor in a clinical setting [1-5]. Furthermore, novel methods by which iatrogenic tumor dissemination can be prevented have been invented for gastric epithelial neoplasia. Inverted laparoscopic and endoscopic cooperative surgery is one of those techniques, in which spillage of gastric juice can be avoided by lifting an intentionally created gastric hole [6]. Nonexposed endoscopic wall-inversion surgery (NEWS) is another technique especially designed for endoscopic local resection of early gastric cancer (EGC) [7-9]. Because the lesion is inverted before endoscopic resection so as not to expose the tumor surface toward the 
peritoneum, EFTR without transgastric access can be realized in the NEWS procedure.

Although these fascinating methods have been developed, there has been no discussion regarding how far a safety margin should be set in determining the resection area. In the Japanese treatment guideline for gastric cancer, $2 \mathrm{~cm}$ should be secured for a safety margin in gastrectomy of EGC [10]. This description, however, is aimed at resection in laparoscopic or open surgery, in which operators have to resect a lesion only in a serosal approach. Because the area of EGC is difficult to demarcate from only the serosal side even though endoscopic clips are placed beforehand, broader resection is imposed in surgery to prevent laterally incomplete resection. In contrast, the endoscopic approach makes possible more accurate and optimized resection under direct visualization of the tumor surface. Accordingly, the resection area can be minimized in EFTR. In endoscopic submucosal dissection of possibly node-negative EGC, it has been empirically accepted that mucosal markings are placed approximately 3-5 mm outside the tumor edge and a mucosal incision is made a few millimeters outside these markings. However, in EFTR of possibly node-positive EGC, neither evidence nor consensus regarding an optimal safety margin exists.

In this study, we aimed to determine the optimal width of the safety margin for EGC which may be a candidate for EFTR by measuring the subepithelial extent (SE) of the lateral tumor margin. Furthermore, we assessed the relationship between the SE and the histological characteristics of the subepithelial tumor edge as well as the possibility to visualize the SE by endoscopic observation.

\section{Patients and methods}

Preparation

After acceptance by the institutional review board of Keio University School of Medicine (approval no. 20130446), we conducted a retrospective analysis in surgically resected submucosal EGCs between January 2009 and June 2013. In the 108 cases, 69 completely resected EGCs $4 \mathrm{~cm}$ or smaller, which could be candidates for NEWS combined with sentinel node navigation surgery as a new concept of minimally invasive gastrectomy of possibly node-positive EGC [11, 12], were selected. Of those, nine cases were excluded owing to insufficient preparation slides or lack of information on how to cut the specimen. Accordingly, 60 submucosal EGCs $4 \mathrm{~cm}$ or smaller with 595 preparation slides were enrolled in this study (Fig. 1). According to the Japanese classification of gastric carcinoma, surgically resected specimens were sliced at 5-mm intervals after being fixed by formalin, and a set of sections were prepared for microscopic observation [13]. In 617 preparation sites stained by hematoxylin and eosin showing the tumor edge, 595 sites which presented lateral tumor borders both on the epithelial surface and on the subepithelial layer were assessed.

Measurement and assessment of the subepithelial extent

Under the supervision of one pathologist (M.S.), two endoscopists (O.G. and A.F.) together measured the SE. Each site of the slides was observed using a microscope with a
Fig. 1 Flowchart of the enrollment in this study. We enrolled 60 submucosal early gastric cancers $4 \mathrm{~cm}$ or smaller for the case-based analysis, and 595 preparation sites showing lateral tumor borders both on the epithelial surface and on the subepithelial layer for the sitebased analysis

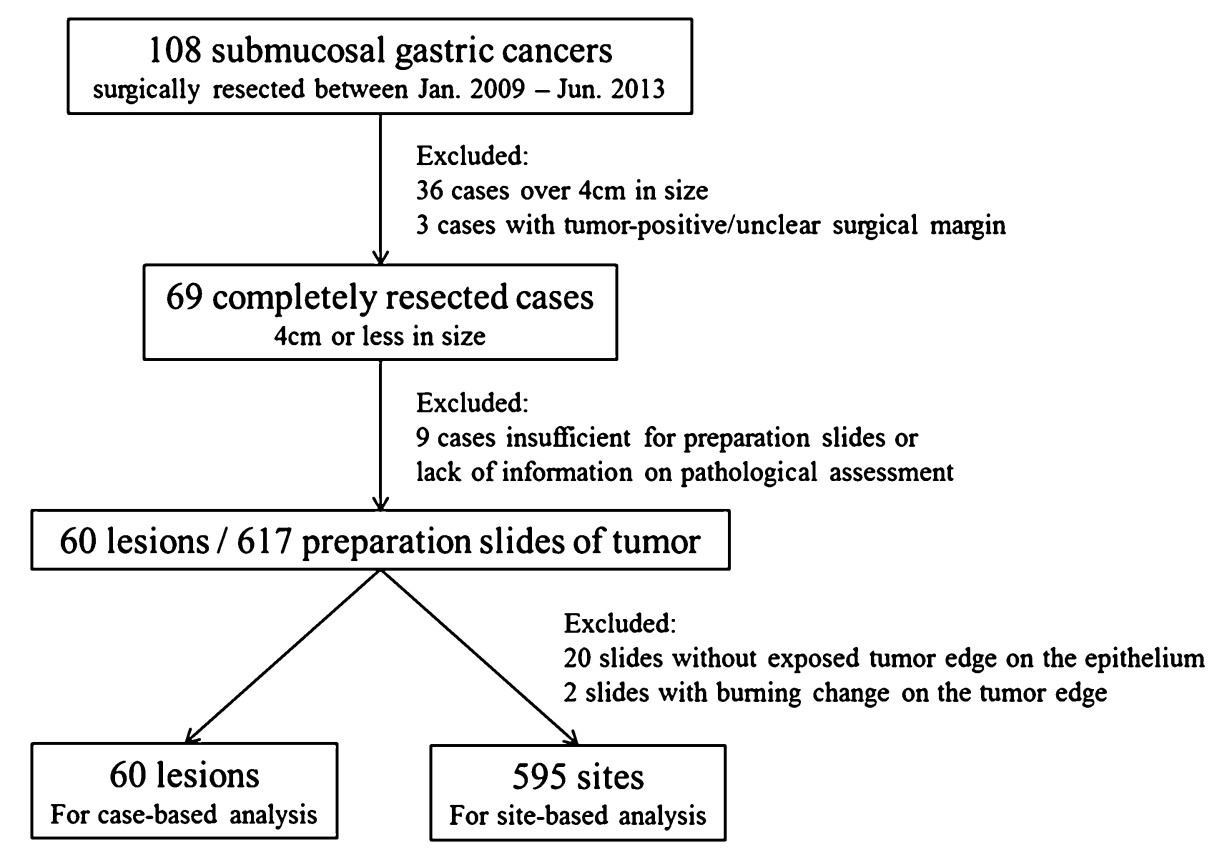


$\times 10$ ocular lens and a $\times 4$ objective lens, and the SE of the lateral tumor margin was measured using an ocular micrometer (U-OCMC10/100XY; Olympus, Tokyo, Japan), by which 39.5 small calibrations were coincident with $1 \mathrm{~mm}$ (one calibration was equal to $25.3 \mu \mathrm{m}$ ). If angiolymphatic invasion was seen outside the primary tumor, the lateral tumor edge was defined as the lateral edge of angiolymphatic invasion. If the tumor edge was exposed to the mucosal surface, the SE was recorded as zero. The method for measuring the SE is shown in Fig. 2. Subsequently, we investigated the correlation between the SE and the following parameters: tumor depth [invasion of the submucosa to a depth of less than $500 \mu \mathrm{m}$ (SM1) or to a depth of at least $500 \mu \mathrm{m}$ (SM2)], histological type (intestinal or diffuse), depth (mucosal or submucosal layer),

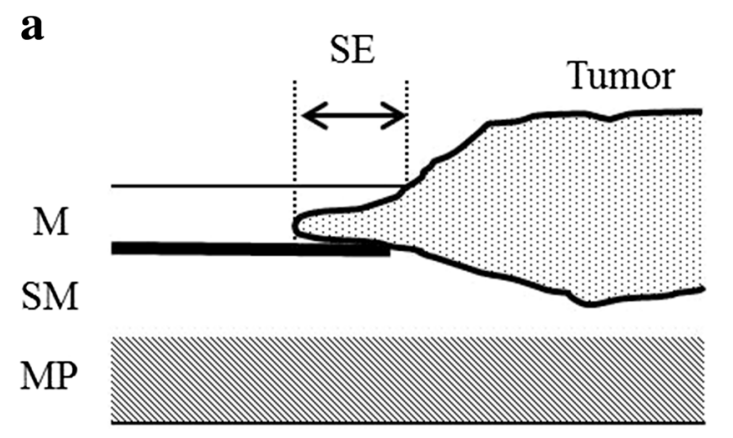

b

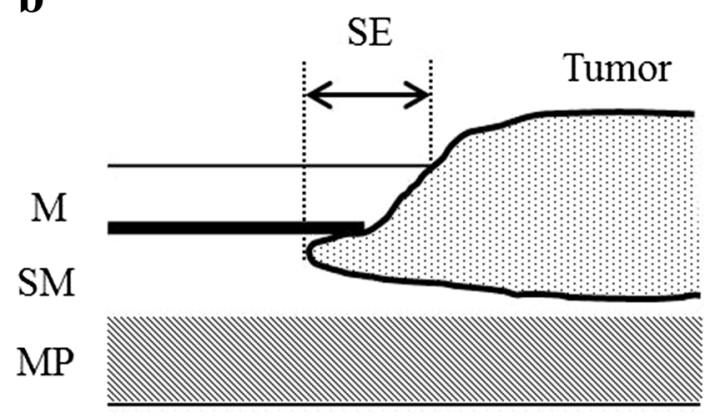

c

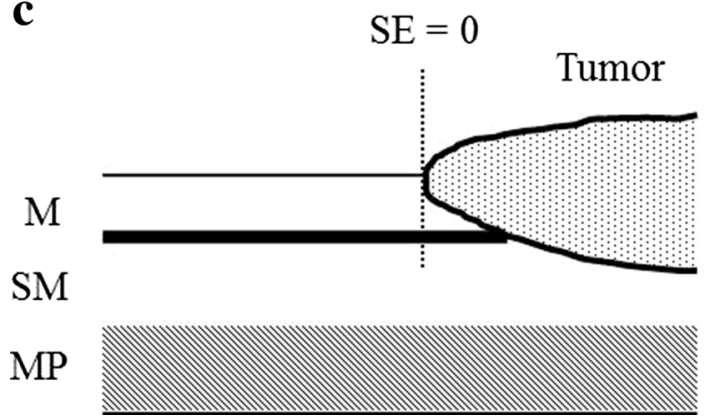

Fig. 2 The method for measuring the subepithelial extent (SE). a The tumor edge is located in the subepithelial mucosal layer. b The tumor edge is spread to the submucosal layer. c The tumor edge is on the mucosal surface. $M$ mucosa, $S M$ submucosa, $M P$ muscularis propria histological characteristics of the tumor edge, and coincidence of histological characteristics between the predominant type and the tumor edge (concordance or discordance). Furthermore, the maximal SE (mSE) in each case was extracted and investigated for a case-based analysis.

Endoscopic estimation of the subepithelial extent

As a subanalysis, we assessed whether endoscopists could estimate the SE in an endoscopic view. Two endoscopists (A.F. and Y.O.) checked still images of the tumor recorded in a preoperative checkup and stored in an electronic patient's record. In each case, approximately 20-30 still images were taken for inspection of the tumor, observed by conventional endoscopy and chromoendoscopy with indigocarmine solution. After checking all images of the tumor, the evaluators estimated the $\mathrm{mSE}(\mathrm{mm})$ and put a mark where the mSE was on the image of the opened and stretched stomach prepared as an answer sheet. In the endoscopic assessment, the evaluators were blind to the results of the histological assessment. The correlation between the endoscopically estimated and the microscopically measured $\mathrm{mSE}$ in each case was investigated. If these two figures were statistically correlated, we planned to assess the geographical concordance of the mSE.

Statistical analysis

We used JMP version 11.0.0 (SAS Institute, Tokyo, Japan) for all statistical analyses. Medians of the SE were compared using the Mann-Whitney $U$ test. The correlation between the endoscopically estimated and the microscopically measured mSE was investigated using Pearson's correlation coefficient. Statistical significance was set at $p<0.05$.

\section{Results}

Clinicopathology information on the enrolled cases is given in Table 1. The numbers of SM1 and SM2 were 14 and 46, respectively. The mean tumor size was $24.4 \mathrm{~mm}$ [standard deviation (SD) $8.5 \mathrm{~mm}$ ]. With regard to the predominant histological type, there were 28 tumors of intestinal type and 32 of diffuse type. A mixed type was present in 50 cases. With regard to the macroscopic type, 11 tumors were elevated and 49 were flat or depressed.

The results of the site-based analysis are shown in Table 2 and Fig. 3. The mean and the median SE were $1.1 \mathrm{~mm}$ (SD $1.8 \mathrm{~mm}$ ) and $0.3 \mathrm{~mm}$ (range $0-12.3 \mathrm{~mm}$ ), respectively. The 99th percentile was $8.8 \mathrm{~mm}$. In the comparison of the SE in the tumor depth, the median SE in 
Table 1 Summary of the clinicopathological features of the cases
$U$ upper third of the stomach, $M$ middle third of the stomach, $L$ lower third of the stomach, $R$ remnant stomach, $A W$ anterior wall, $P W$ posterior wall, $G C$ greater curvature, $L C$ lesser curvature, $S D$ standard deviation, $S M 1$ cancer invading the submucosa to a depth of less than $500 \mu \mathrm{m}, S M 2$ cancer invading the submucosa to a depth of at least $500 \mu \mathrm{m}$

\begin{tabular}{|c|c|}
\hline & Number \\
\hline \multicolumn{2}{|l|}{ Gender } \\
\hline Male & 48 \\
\hline Female & 12 \\
\hline \multicolumn{2}{|l|}{ Location } \\
\hline $\mathrm{U}$ & 10 \\
\hline M & 26 \\
\hline $\mathrm{L}$ & 23 \\
\hline $\mathrm{R}$ & 1 \\
\hline \multicolumn{2}{|l|}{ Circumference } \\
\hline AW & 17 \\
\hline PW & 15 \\
\hline GC & 13 \\
\hline $\mathrm{LC}$ & 15 \\
\hline \multicolumn{2}{|l|}{ Depth $^{\mathrm{c}}$} \\
\hline SM1 & 14 \\
\hline SM2 & 46 \\
\hline \multicolumn{2}{|c|}{ Predominant histological type } \\
\hline Intestinal & 28 \\
\hline Diffuse & 32 \\
\hline \multicolumn{2}{|c|}{ Mixed histological type } \\
\hline Yes & 50 \\
\hline No & 10 \\
\hline \multicolumn{2}{|l|}{ Macroscopic type } \\
\hline Elevated & 11 \\
\hline Flat/depressed & 49 \\
\hline \multicolumn{2}{|c|}{ Angiolymphatic invasion } \\
\hline Yes & 40 \\
\hline \multirow[t]{2}{*}{ No } & 20 \\
\hline & Mean $\pm \mathrm{SD}$ \\
\hline Age (years) & $64.7 \pm 12.4$ \\
\hline Tumor size $(\mathrm{mm})$ & $24.4 \pm 8.5$ \\
\hline
\end{tabular}

446 sites of SM2 was significantly greater than in 149 sites of SM1 ( $0.4 \mathrm{~mm}$ vs $0 \mathrm{~mm}, p=0.0019)$. With regard to the histological type, it was also significantly greater in 297 sites of diffuse-type cancer than in 298 sites of intestinaltype cancer $(0.9 \mathrm{~mm}$ vs $0 \mathrm{~mm}, p<0.0001)$. In the analysis of the depth of the subepithelilal tumor edge, the median SE was greater in 64 sites on the submucosal layer than in 531 sites on the mucosal layer, with a significant difference ( $2.6 \mathrm{~mm}$ vs $0.3 \mathrm{~mm}, p<0.0001$ ). With regard to the histological type at the subepithelilal tumor edge, the median SE was significantly greater in 313 sites of diffuse-type tumor than in 282 sites of intestinal-type tumor $(0.8 \mathrm{~mm}$ vs $0 \mathrm{~mm}, p<0.0001)$. In the analysis of the coincidence of the histological characteristics of the predominant type and the tumor edge, there was no statistical difference in the median SE between 537 sites of concordance and 58 sites of discordance ( $0.3 \mathrm{~mm}$ vs $0.3 \mathrm{~mm}, p=0.65$ ).
Table 2 Site-based analysis of the relationship with the subepithelial extent

\begin{tabular}{|c|c|c|c|}
\hline & Number & $\begin{array}{l}\text { Median subepithelial extent } \\
(\mathrm{mm})\end{array}$ & $p$ \\
\hline Total & 595 & 0.3 (range $0-12.3$ ) & \\
\hline \multicolumn{3}{|l|}{ Depth } & 0.0019 \\
\hline SM1 & 149 & 0 & \\
\hline SM2 & 446 & 0.4 & \\
\hline \multicolumn{3}{|c|}{ Histological type } & $<0.0001$ \\
\hline Intestinal & 298 & 0 & \\
\hline Diffuse & 297 & 0.9 & \\
\hline \multicolumn{3}{|c|}{ Depth of the tumor edge } & $<0.0001$ \\
\hline Mucosa & 531 & 0.3 & \\
\hline Submucosa & 64 & 2.6 & \\
\hline \multicolumn{3}{|c|}{ Histological type of the tumor edge } & $<0.0001$ \\
\hline Intestinal & 282 & 0 & \\
\hline Diffuse & 313 & 0.8 & \\
\hline \multicolumn{3}{|c|}{ Coincidence of histological characteristics } & 0.6484 \\
\hline Concordance & 537 & 0.3 & \\
\hline Discordance & 58 & 0.3 & \\
\hline
\end{tabular}

SM1 cancer invading the submucosa to a depth of less than $500 \mu \mathrm{m}$ $S M 2$ cancer invading the submucosa to a depth of at least $500 \mu \mathrm{m}$

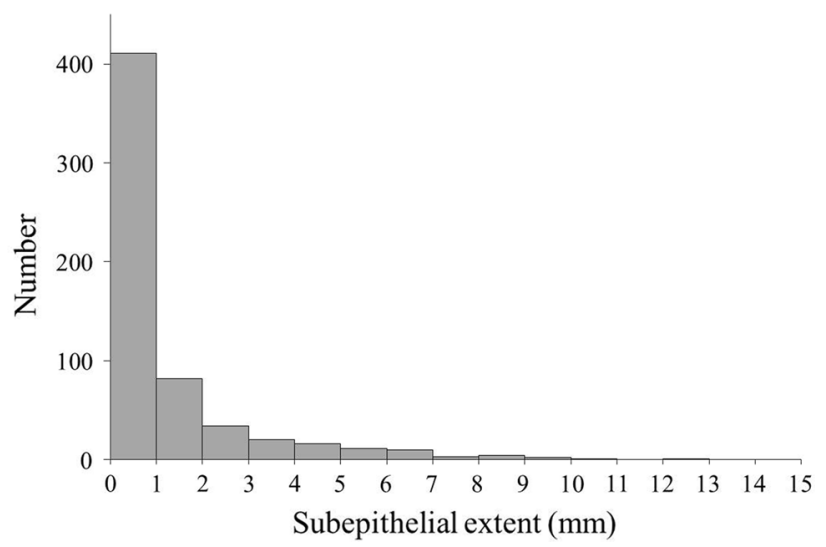

Fig. 3 Distribution of the subepithelial extent. In most sites of the tumor edge, the subepithelial extent is less than $1 \mathrm{~mm}$. The median subepithelial extent is $0.3 \mathrm{~mm}$, and the 99th percentile is $8.8 \mathrm{~mm}$

Table 3 shows the results of the case-based analysis. The mean and the median $\mathrm{mSE}$ were $3.2 \mathrm{~mm}$ (SD $2.9 \mathrm{~mm}$ ) and $2.3 \mathrm{~mm}$ (range $0-12.3 \mathrm{~mm}$ ), respectively. In the comparison of the mSE in the tumor depth, the median mSE in 46 cases of SM2 was greater than in 14 cases of SM1, although no statistical difference existed $(2.4 \mathrm{~mm}$ vs $1.3 \mathrm{~mm}, p=0.16$ ). With regard to the histological type, it was significantly greater in 32 cases of diffuse-type cancer than in 28 cases of intestinal-type cancer $(4.3 \mathrm{~mm}$ vs $1.1 \mathrm{~mm}, p=0.0001)$. With regard to the depth of the subepithelilal tumor edge, the median mSE was greater in 
Table 3 Case-based analysis of the relationship with the maximal subepithelial extent

\begin{tabular}{|c|c|c|c|}
\hline & Number & $\begin{array}{l}\text { Median maximal subepithelial } \\
\text { extent }(\mathrm{mm})\end{array}$ & $p$ \\
\hline Total & 60 & 2.3 (range $0-12.3$ ) & \\
\hline \multicolumn{3}{|l|}{ Depth } & 0.16 \\
\hline SM1 & 14 & 1.3 & \\
\hline SM2 & 46 & 2.4 & \\
\hline \multicolumn{3}{|c|}{ Histological type } & 0.00 \\
\hline Intestinal & 28 & 1.1 & \\
\hline Diffuse & 32 & 4.3 & \\
\hline \multicolumn{3}{|c|}{ Depth of the tumor edge } & 0.00 \\
\hline Mucosa & 48 & 1.7 & \\
\hline Submucosa & 12 & 5.1 & \\
\hline \multicolumn{3}{|c|}{ Histological type of the tumor edge } & 0.00 \\
\hline Intestinal & 26 & 1.0 & \\
\hline Diffuse & 34 & 4.0 & \\
\hline \multicolumn{3}{|c|}{ Coincidence of histological characteristics } & 0.472 \\
\hline Concordance & 52 & 2.4 & \\
\hline Discordance & 8 & 1.8 & \\
\hline
\end{tabular}

SM1 cancer invading the submucosa to a depth of less than $500 \mu \mathrm{m}$ SM2 cancer invading the submucosa to a depth of at least $500 \mu \mathrm{m}$

12 cases on the submucosal layer than in 48 cases on the mucosal layer, with a significant difference $(5.1 \mathrm{~mm}$ vs $1.7 \mathrm{~mm}, p=0.0030$ ). With regard to the histological type at the subepithelilal tumor edge, the median $\mathrm{mSE}$ was also significantly greater in 34 cases of diffuse-type tumor than in 26 cases of intestinal-type tumor $(4.0 \mathrm{~mm}$ vs $1.0 \mathrm{~mm}$, $p=0.0004)$. As with the site-based analysis, in the analysis of the coincidence of the histological characteristics between the predominant type and the tumor edge, we could not find a statistical difference in the median $\mathrm{mSE}$ between 52 cases of concordance and eight cases of discordance ( $2.4 \mathrm{~mm}$ vs $1.8 \mathrm{~mm}, p=0.47$ ). Representative slides of the SE are shown in Fig. 4.

The medians of the endoscopically estimated mSEs which the two endoscopists evaluated were 5.0 and $5.0 \mathrm{~mm}$, with poor correlation $(r=0.39, p=0.0021)$. In the relationship between the endoscopically estimated and the microscopically measured $\mathrm{mSE}$, there were no correlations for both evaluators $(r=-0.027, p=0.84$; and $r=-0.036, p=0.79$, respectively). Accordingly, further assessments for a geographical concordance between the endoscopically estimated and the histologically measured $\mathrm{mSE}$ were not performed.

\section{Discussion}

In this retrospective histology study, we elucidated that the $\mathrm{SE}$ of submucosal EGC $4 \mathrm{~cm}$ or smaller was less than $1 \mathrm{~cm}$ a

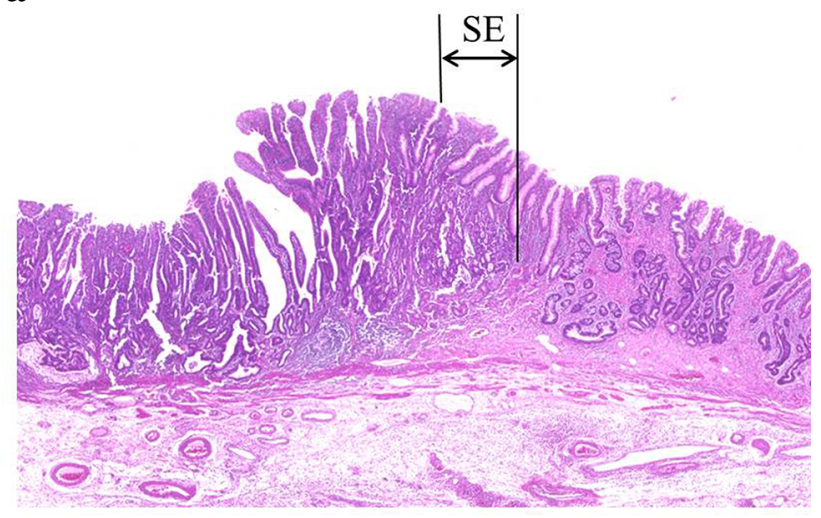

b

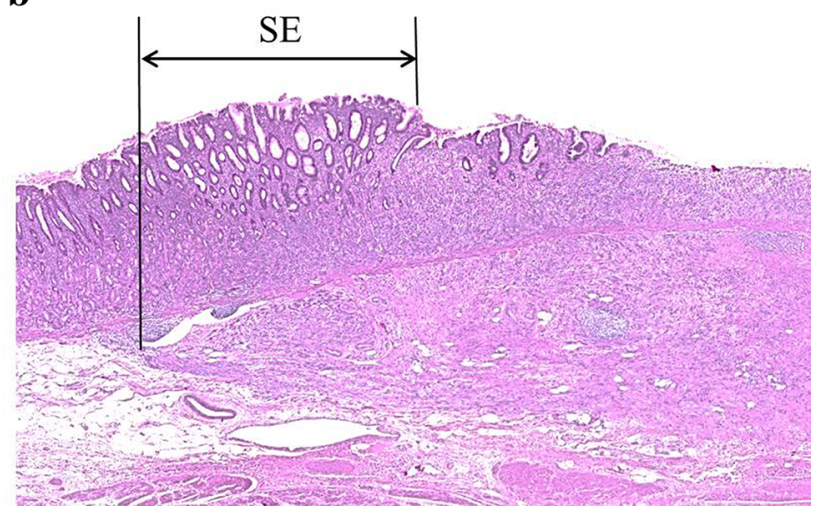

Fig. 4 Representative slides showing the subepithelial extent (SE). a Differentiated cancer is slightly spread in the subepithelial mucosal layer. The subepithelial extent is $0.7 \mathrm{~mm}$. b Diffuse-type cancer invades the submucosal layer laterally. The subepithelial extent is $3.3 \mathrm{~mm}$

in most cases. This study implies that $1 \mathrm{~cm}$ may be sufficient for a lateral safetymargin in EFTR of submucosal EGC $4 \mathrm{~cm}$ or smaller. The results obtained in this study are expected to be helpful for developing techniques of minimally invasive surgery using endoscopy.

We also demonstrated that the SE was greater in SM2 or diffuse-type cancer than in SM1 or intestinal-type cancer. The same tendency was also seen in the analysis with special reference to the histological characteristics of the tumor edge. From those results, we can speculate that submucosal cancer, especially of the diffuse type, is inclined to spread laterally into a deeper layer of the submucosa. This speculation is coincident with the impossibility of estimating the SE by endoscopy because the deeper the cancer invades, the more difficult it is to demarcate the tumor from the surface. Even though the SE might be detected when the invading tumor grows expansively, diffuse-type cancer generally displays infiltrative growth, which may not show a morphological change at the surrounding mucosa of the tumor. In this point, we should be aware of the difference in demarcation of the tumor 
between mucosal cancer in endoscopic submucosal dissection and submucosal cancer in EFTR.

However, we can now clearly detect the exposed cancer edge on the mucosal surface because of the development of diagnostic endoscopy, e.g., narrow band imaging, and magnifying endoscopy, and it is certainly superior in optimizing the resection area to be as small as possible. The results obtained in this study suggest that mucosal markings should be placed $1 \mathrm{~cm}$ outside the exposed tumor edge, which is easily and accurately detected. In clinical settings, keeping a safety margin would be ensured more by cutting the mucosa a few millimeters outside those markings. Practically, some diffuse-type or moderately differentiated EGCs with unexpectedly wider infiltration laterally into the mucosal layer do exist. In these lesions, preoperative biopsy specimens from the surrounding mucosa of the lesion after detection of the endoscopically visualized tumor border would be helpful in order to secure complete resection.

In EFTR, cancer seeding into the peritoneum by intentional perforation of the gastric wall should be avoided. Furthermore, necrosis of the remnant gastric wall might occur after EFTR with standard lymphadenectomy [14]. Accordingly, the area of lymph node dissection should be minimized in order to perform EFTR of possibly nodepositive EGC. In the current situation, NEWS with sentinel node basin dissection is thought to be one of most feasible concepts for minimally invasive gastrectomy among the available methods of surgery for those EGCs [11, 12]. In this technique, if sentinel nodes identified by a radioactive tracer and/or dye solution reveal no metastasis by intraoperative pathological investigation, the area of lymphadenectomy can be limited to a sentinel node basin and, therefore, the resection area of the primary lesion can also be minimized as much as possible [15]. Because NEWS is an EFTR technique without transgastric access, minimally sized local resection can be completed by combining it with sentinel node basin dissection of possibly node-positive EGC. From the results of a large-scale multicenter prospective study, sentinel node navigation surgery may be tentatively applied to EGC for which endoscopic resection is contraindicated (to avoid overinvasiveness) and is $4 \mathrm{~cm}$ or smaller (to avoid a pseudonegative appearance on metastasis) [16]. Therefore, we limited the enrolled cases to sizes of $4 \mathrm{~cm}$ or smaller in this study.

Several limitations exist in the present study. Firstly, this is a retrospective study, although the number of the slides is acceptably large. Secondly, we failed to investigate the lateral edges of the tumor circumferentially because slicing the tumor is mostly unidirectional. Therefore, some slides where a superficially exposed tumor edge was not shown had to be discarded. Thirdly, the endoscopic evaluation was performed using unsystematically stored still images.
To overcome these drawbacks, a prospective study will be required.

In conclusion, the $\mathrm{SE}$ was within $1 \mathrm{~cm}$ in most of nearly 600 sites. In performing EFTR of submucosal EGC $4 \mathrm{~cm}$ or smaller for which minimally invasive gastrectomy can be indicated, one may consider $1 \mathrm{~cm}$ as an acceptable safety margin.

Conflict of interest No conflict of interest exists.

\section{References}

1. Hiki N, Yamamoto Y, Fukunaga T, Yamaguchi T, Nunobe S, Tokunaga $\mathrm{M}$, et al. Laparoscopic and endoscopic cooperative surgery for gastrointestinal stromal tumor dissection. Surg Endosc. 2008;22:1729-35.

2. Abe N, Takeuchi H, Yanagida O, Masaki T, Mori T, Sugiyama $\mathrm{M}$, et al. Endoscopic full-thickness resection with laparoscopic assistance as hybrid NOTES for gastric submucosal tumor. Surg Endosc. 2009;23:1908-13.

3. Zhou PH, Yao LQ, Qin XY, Cai MY, Xu MD, Zhong YS, et al. Endoscopic full-thickness resection without laparoscopic assistance for gastric submucosal tumors originated from the muscularis propria. Surg Endosc. 2011;25:2926-31.

4. Schlag C, Wilhelm D, von Delius S, Feussner H, Meining A. EndoResect study: endoscopic full-thickness resection of gastric subepithelial tumors. Endoscopy. 2013;45:4-11.

5. Mori H, Kobara H, Fujihara S, Nishiyama N, Rafiq K, Oryu M, et al. Feasibility of pure EFTR using an innovative new endoscopic suturing device: the double-arm-bar suturing system (with video). Surg Endosc. 2014;28:683-90.

6. Nunobe S, Hiki N, Gotoda T, Murao T, Haruma K, Matsumoto H, et al. Successful application of laparoscopic and endoscopic cooperative surgery (LECS) for a lateral-spreading mucosal gastric cancer. Gastric Cancer. 2012;15:338-42.

7. Goto O, Mitsui T, Fujishiro M, Wada I, Shimizu N, Seto Y, et al. New method of endoscopic full-thickness resection: a pilot study of non-exposed endoscopic wall-inversion surgery in an ex vivo porcine model. Gastric Cancer. 2011;14:183-7.

8. Mitsui T, Goto O, Shimizu N, Hatao F, Wada I, Niimi K, et al. Novel technique for full-thickness resection of gastric malignancy: feasibility of nonexposed endoscopic wall-inversion surgery (NEWS) in porcine models. Surg Laparosc Endosc Percutan Tech. 2013;23:e217-21.

9. Mitsui T, Niimi K, Yamashita H, Goto O, Aikou S, Hatao F, et al. Non-exposed endoscopic wall-inversion surgery as a novel partial gastrectomy technique. Gastric Cancer. 2014;17:594-9.

10. Japanese Gastric Cancer Association. Japanese gastric cancer treatment guidelines 2010 (ver. 3). Gastric Cancer. 2011; $14: 113-23$.

11. Goto O, Takeuchi H, Kawakubo H, Matsuda S, Kato F, Sasaki M, et al. Feasibility of non-exposed endoscopic wall-inversion surgery with sentinel node basin dissection as a new surgical method for early gastric cancer: a porcine survival study. Gastric Cancer. 2014. doi:10.1007/s10120-014-0358-y.

12. Goto O, Takeuchi H, Kawakubo H, Sasaki M, Matsuda T, Matsuda S, et al. First case of non-exposed endoscopic wall-inversion surgery with sentinel node basin dissection for early gastric cancer. Gastric Cancer. 2014. doi:10.1007/s10120-014-0406-7

13. Japanese Gastric Cancer Association. Japanese classification of gastric carcinoma: 3rd English edition. Gastric Cancer. 2011;14:101-12. 
14. Seto Y, Nakayama H, Ishigami H, Fujii S, Ueda E. An experimental study of intramural blood supply network of the stomach wall. Hepatogastroenterology. 1999;46:2673-6.

15. Takeuchi H, Kitagawa Y. New sentinel node mapping technologies for early gastric cancer. Ann Surg Oncol. 2013;20:522-32.
16. Kitagawa Y, Takeuchi H, Takagi Y, Natsugoe S, Terashima M, Murakami N, et al. Sentinel node mapping for gastric cancer: a prospective multicenter trial in Japan. J Clin Oncol. 2013;31:3704-10. 